\title{
Weak correlations between body height and several brain metrics in healthy elderly subjects
}

\author{
Jäncke, Lutz ; Liem, Franziskus ; Mérillat, Susan
}

\begin{abstract}
The question whether body height is related to different brain size measures has recently gained renewed interest as some studies have reported that body height correlates with intelligence and several brain size measures. In this study, we re-evaluated this question by examining the relationship between body height and different brain size measures including intracranial volume, total brain volume, total cortical surface area, total cortical volume, volume of normal appearing white matter, white matter hyperintensity, cortical surface area, cortical thickness, subcortical gray matter volume, cerebellar cortex and cerebellar white matter in a relatively large sample $(\mathrm{n}=216)$ of physically and cognitively healthy elderly subjects (mean age 71 years, age range $65-85$ years). We identified small correlations $(\mathrm{r}=0.11-$ 0.19) between body height and seven out of ten brain metrics (total brain volume, cortical surface area, cortical volume, subcortical volume, normal appearing white matter volume, and cerebellar gray as well as white matter volumes) when controlling for sex and age. Based on these small relationships between body height and various brain size measures, we discuss the possible reasons and theoretical problems for these small relationships. This article is protected by copyright. All rights reserved.
\end{abstract}

DOI: https://doi.org/10.1111/ejn.14501

Posted at the Zurich Open Repository and Archive, University of Zurich

ZORA URL: https://doi.org/10.5167/uzh-171881

Journal Article

Accepted Version

Originally published at:

Jäncke, Lutz; Liem, Franziskus; Mérillat, Susan (2019). Weak correlations between body height and several brain metrics in healthy elderly subjects. European Journal of Neuroscience, 50(10):3578-3589.

DOI: https://doi.org/10.1111/ejn.14501 
DR. LUTZ JÄNCKE (Orcid ID : 0000-0003-2110-9067)

Article type : Research Report

\section{Weak correlations between body height and several brain metrics in healthy elderly subjects}

Jäncke $^{1 \S}$ L., Liem ${ }^{1,2}$ F., Merillat ${ }^{1,2}$ S.

${ }^{1}$ Division Neuropsychology, Institute of Psychology, University of Zurich, Switzerland

${ }^{2}$ University Research Priority Program (URPP) "Dynamics of Healthy Aging”, University of Zurich, Switzerland

${ }^{\S}$ Corresponding author

\section{Email addresses:}

LJ: lutz.jaencke@uzh.ch

SM: s.merillat@inapic.uzh.ch

FL: franziskus.liem@uzh.ch

\section{Correspondence to:}

Prof. Dr. Lutz Jäncke

Institute of Psychology

Division Neuropsychology

University of Zurich

Binzmühlestrasse 14/25

CH-8050 Zurich, Switzerland

E-mail: lutz.jaencke@uzh.ch

Phone: +41 44635 7400; fax +4144635 7409

Date: 26.2 .2019

This article has been accepted for publication and undergone full peer review but has not been through the copyediting, typesetting, pagination and proofreading process, which may lead to differences between this version and the Version of Record. Please cite this article as doi: 10.1111/ejn.14501

This article is protected by copyright. All rights reserved. 
Short title: Brain size and body height correlation

\begin{abstract}
The question whether body height is related to different brain size measures has recently gained renewed interest as some studies have reported that body height correlates with intelligence and several brain size measures. In this study, we re-evaluated this question by examining the relationship between body height and different brain size measures including intracranial volume, total brain volume, total cortical surface area, total cortical volume, volume of normal appearing white matter, white matter hyperintensity, cortical surface area, cortical thickness, subcortical gray matter volume, cerebellar cortex and cerebellar white matter in a relatively large sample $(n=216)$ of physically and cognitively healthy elderly subjects (mean age 71 years, age range 65-85 years). We identified small correlations ( $\mathrm{r}=$ 0.11 - 0.19) between body height and seven out of ten brain metrics (total brain volume, cortical surface area, cortical volume, subcortical volume, normal appearing white matter volume, and cerebellar gray as well as white matter volumes) when controlling for sex and age. Based on these small relationships between body height and various brain size measures, we discuss the possible reasons and theoretical problems for these small relationships.
\end{abstract}

Keywords Body height, intracranial volume, total brain volume, cortical volume, cortical thickness, cortical surface area, subcortical gray matter, normal appearing cerebral white matter, cerebellar white matter, cerebellum cortex, white matter hyperintensity.

\title{
Introduction
}

The general question of how body parameters such as body size and body weight vary with brain size measures has occupied researchers for some time. While there is ample evidence that brain size varies with body size when using specimen from different taxonomic orders (Jerison, 1979; Roth \& Dicke, 2005), it is currently unclear whether such a relationship exists in humans. Several studies have been conducted in the past using adult human postmortem brains examining brain size/weight and body height relationships. Some studies fail to detect significant correlations whereas others observe significant but relatively weak associations between brain mass and stature (Pakkenberg \& Voigt, 1964; Spann \& Dustmann, 1965;

This article is protected by copyright. All rights reserved. 
Passingham, 1979; Ho et al., 1980; Holloway, 1980; Skullerud, 1985; Garby et al., 1993; Hartmann et al., 1994; Chirachariyavej et al., 2006; Witelson et al., 2006; Heymsfield et al., 2007). However, the most categorical account from these studies has been provided by Haug (1984), declaring that an increase of $10 \mathrm{~cm}$ in body length would be associated with an increase of $60 \mathrm{~g}$ in brain weight. A similar account has been made by Spann and Durstmann (1965), who suggested that each $10-\mathrm{cm}$ body height is related to $83 \mathrm{~g}$ of brain weight in males and $80 \mathrm{~g}$ in females. Using the post-mortem dataset from Pakkenberg and Voigt, Jerison (1979) and Passingham (1979) came to contrasting conclusions, likely because they used different age-cut-off points for subject selection. Using a wider age range (18-45 years), Passingham reported significant correlations, while Jerison noted no significant correlation when using a narrower age range (27-42 years). There are also data available using cranial volume estimates based on the determinations of cranial measures (NASA Reference Publication, 1978 cited from Peters et al. 1998; Jurgens et al., 1990). In these studies, body height and cranial volume correlations are considerably large, ranging from $r=.67$ to $r=.91$.

There are only a few published MRI studies examining brain size and body height relationships. These studies also report heterogeneous results (Willerman et al., 1991; Wickett et al., 1994; Raz et al., 1997; Peters et al., 1998; Nopoulos et al., 2000; Koh et al., 2005; Heymsfield et al., 2009; Taki et al., 2012; Vuoksimaa et al., 2018). The strongest correlation has been reported by Koh and colleagues (2005) on the basis of a 60 young Korean subjects (mean age 21 years). They report an $r=0.57$ for the total sample including men and women. The correlations calculated separately for both sexes are a bit smaller (females: $r=0.47$; males: $r=0.37$ ) but nevertheless significant. Nopoulos and colleagues (2000) report a strong and significant correlation only for women $(r=0.43)$ but not for men $(r$ $=0.02$ ). In the study by Peters et al. (1998) there were also slightly larger (but nonsignificant) correlations for women $(r=0.33$ in sample $S$ and $r=0.21$ in the first sample) while the correlations for men were negligible $(r=0.004$ and $r=0.05)$. The work of Taki and colleagues (Taki et al., 2012) investigated local volume instead of global volume as done in the above-mentioned MRI studies. These authors have calculated the relationship between body height and brain size within a VBM analysis to identify grey matter volume changes in specific brain regions related to body height. In this context, they found significant correlations between the volume of grey matter in the left temporoparietal region, the right prefrontal cortex and the left cerebellum. For these correlations, they only report t-values, 
which we transformed into correlations in order to compare them with the studies presented above. These correlations range between $r=0.31$ (for the left temporoparietal region) and $r=$ 0.44 (for the left cerebellum).

The relationship between body height and brain size has recently received renewed interest, mainly due to the uncovered positive correlation between general cognitive ability (GCA) and body height (Silventoinen et al., 2012; Taki et al., 2012; Keller et al., 2013; Marioni et al., 2014; Adams et al., 2016; Vuoksimaa et al., 2018). This association has been reported for preterm infants (Sammallahti et al., 2014), in the context of aging (Russ et al., 2014), and for a large sample spanning a wide age range (Vuoksimaa et al., 2018). Moreover, the study by Vuoksimaa et al. demonstrated that the positive association between body height and cognitive ability is mediated by brain size.

Body height is highly heritable but is also considered to reflect early life events and is often regarded as a proxy for early brain development. The above-mentioned most recent studies reported brain size and body height relationships ranging between $r=0.1-0.3$ depending on the age range of the study sample and the applied brain size measure. Most of these studies have used total cortical volume or brain volume as a brain measure. Only one study (Vuoksimaa et al., 2018) has simultaneously used total cortical volume and cortical surface area and uncovered moderate brain size and body height relationships (cortical surface area: $r$ $=0.238$; cortical volume: $\mathrm{r}=0.235$ ). To the best of our knowledge, there is no published study available which has used a greater variety of brain measures (e.g., cortical thickness, cortical surface area, intracranial volume, subcortical volume, normal appearing cerebral white matter, cerebellum gray matter, and cerebellum white matter) to shed light on the differential associations with body height. We hypothesize that the different brain measures are differently related to body height.

The present study was therefore designed to examine body height and brain size relationships using a relatively larger set of different brain measures including total intracranial volume, different cortical (cortical thickness, cortical surface area, cortical grey matter volume, cerebral white matter volume), cerebellar (cerebellar white and gray matter volumes), and subcortical measures. Data came from a large sample of healthy older adults $(n=216)$ (mean age $=71$ years). Since this data set comes from a larger longitudinal project and comprises well-educated and healthy subjects, we are explicitly interested to examine whether the reported body height brain size relationships are similar or different compared to previous studies relying on younger samples coming from different ethnic backgrounds.

This article is protected by copyright. All rights reserved. 
Assuming that cortical measures (e.g., cortical volume, surface area, and thickness) are more strongly influenced by use-dependent factors (such as education, skill practice, and diet) (Münte et al., 2002; Pascual-Leone et al., 2005; Jäncke, 2009), the association with body height should be weaker as compared to subcortical volumes. Because intracranial volume is largely independent of use-dependent influences, it is thought to be the most stable brain measure. More specifically, our hypothesis was that intracranial volume would be most strongly related to body height. Owing to the fact that cortical surface area and cortical volume (Luders et al., 2009; Vuoksimaa et al., 2018) is most strongly related to general cognitive ability, we hypothesize that cortical surface area is less stable and will thus only be weakly related to body height.

\section{Methods}

Subjects

Data were taken from the Longitudinal Healthy Aging Brain (LHAB) database - an ongoing project conducted at the University Research Priority Program 'Dynamics of Healthy Aging' of the University of Zurich (Zöllig et al., 2011). We used data from the LHAB baseline assessment which took place between 2011 and 2013. The baseline dataset included 232 subjects $(\mathrm{M}$ age $=70.84 ; 50.43 \%$ female $)$. At each LHAB measurement occasion, the subjects completed an extensive battery of neuropsychological and psychometric cognitive and sensorimotor tests and underwent brain imaging. Eligibility criteria for study participation were age $\geq 64$, a score of $\geq 26$ on the Mini Mental State Examination (MMSE; Folstein et al., 1975), right-handedness (as confirmed by the Annett Handedness Questionnaire (Annett, 1970)), German language proficiency, and no self-report of any neurological or psychiatric disease or other contraindications to MRI. The study was approved by the ethical committee of the canton of Zurich. Participation was voluntary and all subjects gave written informed consent in accordance with the declaration of Helsinki. The data of this sample has been used in previous publications of our group (Madhyastha et al., 2014; Hirsiger et al., 2015; Jäncke et al., 2015; Valizadeh et al., 2017, 2018).

For 216 subjects of the LHAB baseline sample self-reported body height was available (female: $n=109$; male: $n=107$ ). This subsample was used for the subsequent statistical analysis. From these subjects, information about duration of education (in years) was available for 195 subjects. All subjects were well educated and have spent on average approximately 14 years in formal education programs (Table 1). General intelligence was 
measured with a standard German intelligence test (Leistungsprüfsystem: LPS), which is based on Thurstone's intelligence model. Here we used the version for subjects older than 50 years up to 90 years (Sturm et al., 1993). The split-half reliability of this test ranges between $\mathrm{r}$ $=.89$ and $\mathrm{r}=.97$ depending on the particular subtest. The detailed data for age, body height, years of education, and IQ are shown in Table 1.

\section{Image Acquisition}

MRI data were acquired with a 3.0T Philips Ingenia scanner (Philips Medical Systems, Best, The Netherlands). We have described the image acquisition procedure in several of our recent papers, thus we partly reiterate what we have mentioned in these papers (Madhyastha et al., 2014; Hirsiger et al., 2015; Jäncke et al., 2015; Valizadeh et al., 2018). T1-weighted images were recorded with a gradient echo sequence (3D turbo field echo, 160 sagittal slices, slice thickness $=1 \mathrm{~mm}$, in-plane resolution $=1 \times 1 \mathrm{~mm}, \mathrm{FOV}=240 \times 240 \mathrm{~mm}$, repetition time $=8.18 \mathrm{~ms}$, echo time $=3.80 \mathrm{~ms}$, flip angle $=8^{\circ}$ ). FreeSurfer (v5.3) was used to obtain measurements of cortical and subcortical anatomy (Fischl et al., 2002; Fischl, Salat, et al., 2004; Fischl, van der Kouwe, et al., 2004; Destrieux et al., 2010). All MRI measurements have been subjected to precise quality control before statistical analysis. After completing the standard recon-all pipeline, measurements for cortical thickness, surface area, and volume were extracted for the regions of the Destrieux (aparc.a2009s) parcellation scheme (Destrieux et al., 2010). Subcortical and global volume measurements were also extracted from FreeSurfer's aseg segmentation. This procedure revealed the following anatomical measures:

(1) Intracranial volume (ICV).

(2) Total brain volume without ventricles, CSF, and choroid plexus (BV).

(3) Mean cortical thickness (CT).

(4) Total cortical surface area (CSA).

(5) Total cortical grey matter volume (CortexVol), which is the volume inside the pial surface minus the volume inside the white surface minus tissue inside the ribbon that is not part of cortex (eg, hippocampus).

(6) Total subcortical gray matter volume (SubCorticalGrayVol) includes thalamus, caudate, putamen, pallidum, hippocampus, amygdala, accumbens, ventral DC, substantia nigra (if there).

(7) Total cerebral normal appearing white matter volume (NAWM), which is volume inside 
the white surface minus anything that is not WM. It does not include cerebellar white matter or brainstem. The volume of the white matter hyperintensities (WMH) are subtracted from the cerebral white matter count.

(8) Total volume of white matter hyperintensities (WMH).

(9) Total cerebellar gray matter volume (CBGM).

(10) Total cerebellar white matter volume (CBWM).

We have chosen to work with these brain metrics because they scale differently to brain size (Jäncke et al., 2019).

\section{Statistical analysis}

We conducted several steps for statistical analysis:

(1) Calculation of means and standard deviations for each brain measure broken down for sex.

(2) Calculation of intercorrelations and factor analysis between the different brain metrics.

(3) Multiple regressions with body height $(\mathrm{BH})$, Sex, the interaction between $\mathrm{BH}$ and Sex (Sex:BH), BH and Age (BH:Age), and Age and Sex (Age:Sex) as independent variables separately for each brain metric. We excluded the threefold interaction between $\mathrm{BH}$, Sex, and Age because these interactions are difficult to interpret and the relaimpo software (described below) cannot process threefold interactions.

(4) In order to estimate the relative importance of each predictor in explaining the variability of the particular brain metrics, we used the relaimpo package provided in the context of the $\mathrm{R}$ software (R Core Team, 2013). Here we used the $l m g$ metric providing a decomposition of the model explained variance into non-negative contributions of each predictor. While the assessment of relative importance in linear models is simple, as long as all regressors are uncorrelated, it becomes more problematic as the predictors are correlated (as in our case), so that it is no longer straightforward to break down the $\mathrm{R}^{2}$ values into shares from the individual regressors. The here used $l m g$ metric is a valid estimator, which has been proposed for such cases (Grömping, 2006).

(5) Based on the results of the afore-mentioned regressions, a series of linear correlations between $\mathrm{BH}$ and each brain metric (corrected for Age and Sex) were computed.

This article is protected by copyright. All rights reserved. 
(6) For graphic description, scatterplots between BH (corrected for Age and Sex) are plotted.

Using a relatively large sample as in our study, even small effects (correlations) could become significant. We therefore used $r^{2}, r$, and Cohen's $d$ values as indicators of effect sizes. These effect sizes were evaluated according to Cohen's suggestion (Cohen, 1992) (for $r$ values: small: $r=0.1-0.3$; medium: $r>0.3-0.5$; large: $r>0.5$; for $r^{2}$ values: small: $r^{2}=0.01$ - 0.09 [1-9\%]; medium: $\mathrm{r}^{2}>0.09$ - 0.25 [9-25\%]; large: $\mathrm{r}^{2}>0.25$ [>25\%]; for Cohen's d: small: $d=0.2-0.5$; medium: $d=0.5-0.8$; large: $d>0.8)$. In addition, exact $p$ values are reported since $\mathrm{p}$ values can also be used as effect size measures (Krauth, 1988). The results of our analyses are not interpreted in terms of statistical significance. The p-value is defined as the lowest significance level at which one would still have obtained a significant result for a given data set, a given significance test, and a given test problem. This has the advantage that other researchers can decide for themselves whether the results are significant at the significance level they find acceptable. All statistical analyses were conducted with routines from the $\mathrm{R}$ software (R Core Team, 2013) on a MacBook Pro. We will focus mainly on results associated with small and/or medium effect sizes.

\section{Results}

Table 1 demonstrates the descriptive data for our sample. Age is similar for both sexes, while body height, years of education, and IQ substantially differ between both sexes. As expected, women are substantially smaller than men (mean body height \pm s.d.; women $=164.7 \mathrm{~cm} \pm 5.9$ $\mathrm{cm}$; men: $175.9 \mathrm{~cm} \pm 6 \mathrm{~cm}$; $\mathrm{d}=1.89)$. Men demonstrate on average more years of formal education ( women $=13.4$ years \pm 3 years; men: 15.8 years \pm 3.8 years; $d=0.67$ ). In addition, men performed moderately superior in the IQ test (women $=119.7 \pm 5.9 ;$ men: $122 \pm 7.3$; $\mathrm{d}=$ 0.48). The slightly higher IQ in men could possibly be due to the longer education in men which might have increased the IQ. In fact, the correlation between IQ and years of education is moderately high $\left(\mathrm{r}=0.234 ; \mathrm{CI}_{95 \%}: 0.096-0.364 ; \mathrm{p}=0.001064\right)$.

Table 2 shows the means and standard deviations for the brain metrics broken down for sex. These measures are roughly similar to those reported in previous studies on subjects from the same age range (Jäncke et al., 2015). For most brain metrics men demonstrate larger values except for CT and WMH. The sex differences for CerebellumWhiteMatter volume was of medium size $(d=0.34)$.

This article is protected by copyright. All rights reserved. 
Table 3 shows the intercorrelations between the different brain metrics. For these brain metrics a factor analysis with subsequent varimax rotation was performed revealing six independent factors explaining 96.1 of the variance. The results of this factor analysis are demonstrated in Table 4. Factor 1 explaining $44.1 \%$ of the variance represents most of the brain metrics (BV, CSA, SubCortGrayVol, and NAWM). Factors 2-6 representing one brain metrics each (factor 2: CerebellumWhiteMatter, factor 3: CT; factor 4: WMH; factor 5: ICV, factor 6: CerebellumCortex).

In Table 5 the results of the multiple regression analyses with the brain metrics as dependent variables and BH, Sex, Age, Sex:Age, BH:Sex., and BH:Age are summarized. Shown are the percentages of explained variances for all independent variables as provided by the relaimpo and the $\operatorname{lmg}$ procedures. As can be seen from this Table, Sex, Age, and BH explain most of the variance in nearly all brain metrics. The interactions (Sex:Age, BH:Age, and BH:Sex) explain less than $1 \%$ of the variance and thus are deemed as unimportant for the further analysis. Subsequently performed bootstrap analysis revealed that Sex explained most of the variance for nearly all brain metrics (ICV, BV, CSA, CortexVol, SubCortGrayVol, and NAWM). The effect sizes in terms of explained variance $\left(r^{2} * 100\right)$ are of medium size for the above-mentioned brain metrics. Age is most important for CT (11.9\% of explained variance) and WMH (15.5\% of explained variance) with medium effect sizes. Small Age influences are found for ICV and CSA. Moderately strong influences of BH are found for BV, CSA, CortexVol, and NAWM. Small BH influences are found for ICV and SubCortGrayVol. BH exerted no influence on CT and WMH.

Based on results of the above-mentioned regression analysis all brain metrics were linearly corrected for Age and Sex. With these corrected brain metrics, Pearson correlations were computed with $\mathrm{BH}$. The results of the correlations with $\mathrm{BH}$ are shown in Table 6. As can be seen in Table 6, there are several small correlations $(\mathrm{r}=0.1-0.3)$ between $\mathrm{BH}$ and some brain metrics (BV, CSA, CortexVol, SubCortGrayVol, NAWM, CerebellumCortex, and CerebellumWhiteMatter). The largest correlations were found for BV ( $\mathrm{r}=0.19, \mathrm{CI}_{95 \%}: 0.06$ $0.31)$ and Cortex Vol $\left(\mathrm{r}=0.19, \mathrm{CI}_{95 \%}: 0.06-0.31\right)$. The scatterplots between $\mathrm{BH}$ and the brain metrics (corrected for Age and Sex) are depicted in Figure 1.

This article is protected by copyright. All rights reserved. 
Because body height has repeatedly been related to general cognitive ability, we also computed correlations between psychometric intelligence and body height (corrected for Sex and Age) revealing practically no correlation $\left(\mathrm{r}=-0.007, \mathrm{CI}_{95 \%}:-0.14-0.12\right.$, df: $209, \mathrm{p}=$ 0.9151).

For the sake of completeness, we checked whether there are also non-linear relationships between $\mathrm{BH}$ and the brain measures. However, we did not find any non-linear relationship either by visual inspection or by including non-linear terms to the multiple regressions.

\section{Discussion}

The goal of this paper was to examine the relationship between body height and brain anatomical measures in a relatively large group of healthy older subjects. This question has recently come back into focus because several studies have reported a moderate body height to brain size relationship in preterm children (Sammallahti et al., 2014), in the context of aging (Russ et al., 2014), and in large samples of mostly younger subjects (Posthuma et al., 2000; Vuoksimaa et al., 2018). Furthermore, this relationship has been associated with cognitive ability, in a way that brain size mediates the association between body height and cognitive ability (Vuoksimaa et al., 2018).

In our study, we identified small relationships between several brain size metrics (BV, CSA, CortexVol, SubCortVol, NAWM, CerebellumCortex, and CerbellumWhiteMatter) and body height. These correlations ranged between $r=0.11$ to $r=0.19$ and are small according to the effect size categorization proposed by Cohen (1992). Thus, body height only accounts for approximately $1-4 \%$ of the entire variance of these brain metrics. These brain size and body height relationships are smaller than those reported in most of the previously published papers using different samples and brain measures (Willerman et al., 1991; Wickett et al., 1994; Raz et al., 1997; Peters et al., 1998; Nopoulos et al., 2000; Posthuma et al., 2000; Koh et al., 2005; Heymsfield et al., 2009; Taki et al., 2012; Vuoksimaa et al., 2018).

The brain size body height relationships we identified in our sample are close to those reported by Vuoksimaa and colleagues (2018) and Posthuma and colleagues (2000). Vuoksimaa et al. calculated correlations between body height and cortical volume and cortical surface area in a large $(n=534)$ middle-aged (51-60 years old) sample of men from the Vietnam Era Twin Registry containing 131 monozygotic (MZ) and 96 dizygotic (DZ) twins. For segmentation of cortical volume and cortical surface area they have used the same 
freesurfer procedure as we have applied in our study. They identified slightly larger correlations than we have identified in our study (body height with cortical volume: $\mathrm{r}_{\text {Vuoksimaa }}$ $=0.235, r_{\text {present study }}=0.19$; body height with cortical surface area: $r_{\text {Vuoksimaa }}=0.238, r_{\text {present }}$ study $=0.16$ ). Possible reasons for these differences are difficult to identify. Whether differences in terms of ethnicity, age range, IQ, or the fact that twins have been studied could account for the slightly higher correlations, are currently more or less speculative. An older study by Posthuma et al. (2000) calculated correlations between body height and intracranial volume as well as cerebellar volume in 256 younger subjects (mean age approximately 30 years). Most of these subjects are either MZ or DZ twins from the Netherlands Twin Registry. The brain metrics used in this study were estimated on the basis of a semi-automatic in-house procedure, for which it is unclear whether this procedure results in the same intracranial and cerebellar volume estimates as the freesurfer protocol we and Vuoksimaa and colleagues (2018) have used. However, the reported correlations are partly similar to those we have obtained in our study for similar brain metrics. For their intracranial volume measurement they obtained small (but significant) correlations (men: r=0.194; women: $\mathrm{r}=0.229$ ). In our study, we did not find even a small correlation between body height and intracranial volume $(\mathrm{r}=-0.06)$. The correlations between body height and cerebellar volume (men: $r=0.280$; women: $r=0.194$ ) are more similar to ours (CerebellumCortex: $r=0.15$; CerbellumWhiteMatter: $r=0.11$ ) although numerically smaller. Similarly, as for the findings from Vuoksimaa and colleagues (2018) it is difficult to explain the differences between the correlations from our and their study. Methodological differences in estimating the brain metrics are most likely not responsible since both studies have used the same methods for measuring the brain compartments. However, the differences of the used samples are quite drastic in terms of age and IQ. In terms of IQ the subjects of the Zurich sample demonstrate above average IQ scores. Whether this might be responsible for the between-study differences is difficult to explain. In addition, the Zurich sample comprises older subjects while the sample of Posthuma et al. (2000) comprises much younger subjects with a mean age of approximately 30 years. Thus, it might be that some age-related brain changes have occurred in the subjects of our sample. However, the intracranial volume should be stable across the entire life span. Thus, aging could not account entirely for the apparent differences. However, despite the different correlation for body height and ICV, the correlations for cerebellar volumes are quite similar although still small.

This article is protected by copyright. All rights reserved. 
Most of the brain metrics we have used in our study load on one factor (BV, CSA, CortexVol, SubCortGrayVol, and NAWM). For these brain metrics small correlations with body height were obtained $(\mathrm{r}=0.11-0.19)$. For CerebellumCortex (loading on factor 6) and CerbellumWhiteMatter (loading on factor 2) there were also small correlations with body height $(r=0.11-0.15)$. The other brain metrics were either not related to body height (ICV loading on factor 5; WMH loading on factor 4) or did not pass the threshold of a "small" effect ( $\mathrm{r}=0.1$ ) (CT loading on factor 3, $\mathrm{r}=0.09$ ). Thus, the relationship to body height is not similar for all brain regions. This result partly conforms with the results of the study by Taki and colleagues (2012) who reported regional specific correlations with body height in the context of a VBM analysis with moderate correlations ( $\mathrm{r}=0.31-0.44)$ between body height and grey matter volumes in the left temporoparietal region, the right prefrontal cortex and the left cerebellum. This comes as no surprise since it is meanwhile known that the different brain compartments scale differently to brain size (Jäncke et al., 1997, 2019; Lüders et al., 2002; Reardon et al., 2018). Although the correlations between total brain volume and the volumes of different brain compartments (e.g., NAWM, SubCortGrayVol) are strongly related with each other, they scale differently to total brain size. For example, cerebral WM and the accumbens volumes scaled out of proportion with total brain volume, whereas most other brain compartment measures scaled less than proportional to brain size. Thus, larger brains exhibit relatively larger cerebral NAWM volumes than do smaller brains. Cortical gray matter (and most other brain compartmental measures), on the other hand, relatively decreases as total brain volume increases.

Although body size and brain size are strongly influenced by genetic factors (Widdowson, 1951; Paigen et al., 1987; Posthuma et al., 2000; Lai, 2006; Sudfeld, McCoy, Danaei, et al., 2015; Sudfeld, McCoy, Fink, et al., 2015; Lukies et al., 2017), they differ in several respects, which most likely mitigate brain size and body height correlations. First of all, the lifespan trajectories of both measures are different. Brain size changes are roughly following an inverted U-shaped function with a strong increase of brain volume during the first 18 years of life followed by a relatively stable phase until the age of 60 (for most brain measures, some are changing even a bit earlier), after which brain size decreases (Hedman et al., 2012; Ziegler et al., 2012; Valizadeh et al., 2017). Body height, on the other hand, is more or less stable during adulthood. Thus, it is unlikely that the relationship between body height and brain size measures are stable over the entirely life span. Secondly, the individual aging trajectories of the brain measures are shaped by many different factors. Preclinical signs of 
dementia, for example, may impact on the brain measures and may lead to earlier or accelerated decline although cognition is still normal (Costafreda et al., 2011; Gaser et al., 2013). Stress or unfavorable genetic configurations (e.g., genes coding pathologies like Huntington's disease or Down syndrome) may be other factors in determining brain structural decline (Mattson et al., 2002; Bhatt et al., 2018; Miskolczi et al., 2018). Most importantly, however, is the possibility that the usage and non-usage of specific psychological and motor functions have an influence on brain size. Many previous studies have shown that specific brain parts are considerably influenced by practice and non-use (Münte et al., 2002; Jäncke, 2009; Voelcker-Rehage et al., 2010; Erickson et al., 2011; Zatorre et al., 2012; Voss et al., 2013; Oltmanns et al., 2017). Taken together, there are more sources of variance for the brain size metrics obtained in adulthood and during aging than for body height, possibly mitigating brain size and body height correlations.

In sum, our findings do not support the existence of a strong or even moderate relationship between body height and brain size (and other brain metrics). We identified rather small relationships between some of our brain metrics and body height. This result has considerable consequences for all neuroanatomical studies in which body height is taken into account. If there is not a strong relationship between body height and brain size, the former is not an appropriate variable for the normalization of brain metrics in order to control for sex differences. Intracranial volume or total brain volume may be the more appropriate control measures (e.g., Jäncke et al., 2015). More importantly is, however, that the body size and brain size relationship is far from being understood. As mentioned in our earlier paper 20 years ago (Peters et al., 1998), there are still "unsolved problems in comparing brain sizes in Homo sapiens". If body height and brain size correlations are calculated using heterogeneous samples in terms of ethnicity, sex, health status or age, then these correlations may well be moderate. However, for homogeneous samples (for example, if these correlations are calculated only within one ethnic or one sex group), the correlations usually remain weak and insignificant. Currently, we do not know how ethnicity and sex are genetically coded to induce different body heights and brain sizes. For example, Yemenitic bodies (which have been identified as those ethnic groups with the smallest body height) are not simply scaleddown versions of the bodies of the Dutch (who have been identified as those ethnic groups with the largest body height) (NCD Risk Factor Collaboration (NCD-RisC), 2016). The same pertains for brain size measures for which we cannot assume that smaller brains are simply linearly scaled down versions of larger brains. It becomes more and more clear that large and 
small brains differ not only in brain size but also in other anatomical (e.g., connectivity, white matter volume, or corpus callosum size) (Ringo et al., 1994; Jäncke et al., 1997, 2019; Lüders et al., 2002; Luders et al., 2004, 2005, 2006; Hänggi et al., 2014; Kurth et al., 2017) and functional features (Jäncke \& Steinmetz, 1998).

\section{Acknowledgment}

The current analysis incorporates data from the Longitudinal Healthy Aging Brain (LHAB) database project, which is carried out as one of the core projects at the International Normal Aging and Plasticity Imaging Center / INAPIC and the University Research Priority Program "Dynamics of Healthy Aging" of the University of Zurich. The following members of the core INAPIC team were involved in the design, set-up, maintenance, and support of the LHAB database: Anne Eschen, Lutz Jäncke, Mike Martin, Susan Merillat, Christina Rocke, and Jacqueline Zöllig.

\section{Statement regarding potential conflicts of interest}

The authors have no conflict of interest to declare.

\section{Author contributions}

Lutz Jäncke: supervision of data acquisition, experimental design, data analysis, writing of the manuscript

Franziskus Liem: supervision of data acquisition, data analysis, reviewing of the manuscript.

Susan Merillat: supervision of data acquisition, data analysis, reviewing of the manuscript.

\section{Data accessibility}

The dataset analyzed for this study is available from the corresponding author on reasonable request.

This article is protected by copyright. All rights reserved. 


\section{Abbreviations}

BH: body height

BV: Total brain volume without ventricles, CSF, and choroid plexus

CBGM: Total cerebellar gray matter volume

CBWM: Total cerebellar white matter volume

CerebellumCortex: Total cerebellar gray matter volume

CerebellumWhiteMatter: Total cerebellar white matter volume

$\mathrm{CI}_{95 \%}: 95 \%$ confidence interval

cm: centimeter

CortexVol: Total cortical grey matter volume

CSA: Total cortical surface area

CT: Mean cortical thickness

ICV: Intracranial volume

FOV: field of view

IQ: psychometric intelligence

1HAB: Longitudinal Healthy Aging Brain

LPS: Leistungsprüfsystem

MMSE: Mini Mental State Examination

MRI: magnetic resonance imaging

NAWM: Total cerebral normal appearing white matter volume

P: probability

$\mathrm{R}$ : correlation

$\mathrm{R}^{2}$ : percent of explained variance

Sd: standard deviation

SubCorticalGrayVol: Total subcortical gray matter volume

SBCOGM: Total subcortical gray matter volume

This article is protected by copyright. All rights reserved. 
WMH: Total volume of white matter hyperintensities

\section{ORCID}

https://orcid.org/0000-0003-2110-9067

\section{References}

Adams, H.H.H., Hibar, D.P., Chouraki, V., Stein, J.L., Nyquist, P.A., Rentería, M.E., Trompet, S., Arias-Vasquez, A., Seshadri, S., Desrivières, S., Beecham, A.H., Jahanshad, N., Wittfeld, K., Van der Lee, S.J., Abramovic, L., Alhusaini, S., Amin, N., Andersson, M., Arfanakis, K., Aribisala, B.S., Armstrong, N.J., Athanasiu, L., Axelsson, T., Beiser, A., Bernard, M., Bis, J.C., Blanken, L.M.E., Blanton, S.H., Bohlken, M.M., Boks, M.P., Bralten, J., Brickman, A.M., Carmichael, O., Chakravarty, M.M., Chauhan, G., Chen, Q., Ching, C.R.K., Cuellar-Partida, G., Braber, A.D., Doan, N.T., Ehrlich, S., Filippi, I., Ge, T., Giddaluru, S., Goldman, A.L., Gottesman, R.F., Greven, C.U., Grimm, O., Griswold, M.E., Guadalupe, T., Hass, J., Haukvik, U.K., Hilal, S., Hofer, E., Hoehn, D., Holmes, A.J., Hoogman, M., Janowitz, D., Jia, T., Kasperaviciute, D., Kim, S., Klein, M., Kraemer, B., Lee, P.H., Liao, J., Liewald, D.C.M., Lopez, L.M., Luciano, M., Macare, C., Marquand, A., Matarin, M., Mather, K.A., Mattheisen, M., Mazoyer, B., McKay, D.R., McWhirter, R., Milaneschi, Y., Mirza-Schreiber, N., Muetzel, R.L., Maniega, S.M., Nho, K., Nugent, A.C., Loohuis, L.M.O., Oosterlaan, J., Papmeyer, M., Pappa, I., Pirpamer, L., Pudas, S., Pütz, B., Rajan, K.B., Ramasamy, A., Richards, J.S., Risacher, S.L., Roiz-Santiañez, R., Rommelse, N., Rose, E.J., Royle, N.A., Rundek, T., Sämann, P.G., Satizabal, C.L., Schmaal, L., Schork, A.J., Shen, L., Shin, J., Shumskaya, E., Smith, A.V., Sprooten, E., Strike, L.T., Teumer, A., Thomson, R., TordesillasGutierrez, D., Toro, R., Trabzuni, D., Vaidya, D., Van der Grond, J., Van der Meer, D., Van Donkelaar, M.M.J., Van Eijk, K.R., Van Erp, T.G.M., Van Rooij, D., Walton, E., Westlye, L.T., Whelan, C.D., Windham, B.G., Winkler, A.M., Woldehawariat, G., Wolf, C., Wolfers, T., Xu, B., Yanek, L.R., Yang, J., Zijdenbos, A., Zwiers, M.P., Agartz, I., Aggarwal, N.T., Almasy, L., Ames, D., Amouyel, P., Andreassen, O.A., Arepalli, S., Assareh, A.A., Barral, S., Bastin, M.E., Becker, D.M., Becker, J.T., Bennett, D.A., Blangero, J., van Bokhoven, H., Boomsma, D.I., Brodaty, H., Brouwer, R.M., Brunner, H.G., Buckner, R.L., Buitelaar, J.K., Bulayeva, K.B., Cahn, W., Calhoun, V.D., Cannon, D.M., Cavalleri, G.L., Chen, C., Cheng, C.-Y., Cichon, S., Cookson, M.R., Corvin, A., Crespo-Facorro, B., Curran, J.E., Czisch, M., Dale, A.M., Davies, G.E., De Geus, E.J.C., De Jager, P.L., de Zubicaray, G.I., Delanty, N., Depondt, C., DeStefano, A.L., Dillman, A., Djurovic, S., Donohoe, G., Drevets, W.C., Duggirala, R., Dyer, T.D., Erk, S., Espeseth, T., Evans, D.A., Fedko, I.O., Fernández, G., Ferrucci, L., Fisher, S.E., Fleischman, D.A., Ford, I., Foroud, T.M., Fox, P.T., Francks, C., Fukunaga, M., Gibbs, J.R., Glahn, D.C., Gollub, R.L., Göring, H.H.H., Grabe, H.J., Green, R.C., Gruber, O., Gudnason, V., Guelfi, S., Hansell, N.K., Hardy, J., Hartman, C.A., Hashimoto, R., Hegenscheid, K., Heinz, A., Le Hellard, S., Hernandez, D.G., Heslenfeld, D.J., Ho, B.C., Hoekstra, P.J., Hoffmann, W., Hofman, A., Holsboer, F., Homuth, G., Hosten, N., Hottenga, J.-J., Hulshoff Pol, H.E., Ikeda, M., Ikram, M.K., Jack, C.R., Jr, Jenkinson, 
M., Johnson, R., Jönsson, E.G., Jukema, J.W., Kahn, R.S., Kanai, R., Kloszewska, I., Knopman, D.S., Kochunov, P., Kwok, J.B., Lawrie, S.M., Lemaître, H., Liu, X., Longo, D.L., Longstreth, W.T., Jr, Lopez, O.L., Lovestone, S., Martinez, O., Martinot, J.-L., Mattay, V.S., McDonald, C., McIntosh, A.M., McMahon, K.L., McMahon, F.J., Mecocci, P., Melle, I., Meyer-Lindenberg, A., Mohnke, S., Montgomery, G.W., Morris, D.W., Mosley, T.H., Mühleisen, T.W., Müller-Myhsok, B., Nalls, M.A., Nauck, M., Nichols, T.E., Niessen, W.J., Nöthen, M.M., Nyberg, L., Ohi, K., Olvera, R.L., Ophoff, R.A., Pandolfo, M., Paus, T., Pausova, Z., Penninx, B.W.J.H., Pike, G.B., Potkin, S.G., Psaty, B.M., Reppermund, S., Rietschel, M., Roffman, J.L., Romanczuk-Seiferth, N., Rotter, J.I., Ryten, M., Sacco, R.L., Sachdev, P.S., Saykin, A.J., Schmidt, R., Schofield, P.R., Sigurdsson, S., Simmons, A., Singleton, A., Sisodiya, S.M., Smith, C., Smoller, J.W., Soininen, H., Srikanth, V., Steen, V.M., Stott, D.J., Sussmann, J.E., Thalamuthu, A., Tiemeier, H., Toga, A.W., Traynor, B.J., Troncoso, J., Turner, J.A., Tzourio, C., Uitterlinden, A.G., Hernández, M.C.V., Van der Brug, M., Van der Lugt, A., Van der Wee, N.J.A., Van Duijn, C.M., Van Haren, N.E.M., Van T Ent, D., Van Tol, M.-J., Vardarajan, B.N., Veltman, D.J., Vernooij, M.W., Völzke, H., Walter, H., Wardlaw, J.M., Wassink, T.H., Weale, M.E., Weinberger, D.R., Weiner, M.W., Wen, W., Westman, E., White, T., Wong, T.Y., Wright, C.B., Zielke, H.R., Zonderman, A.B., Deary, I.J., DeCarli, C., Schmidt, H., Martin, N.G., De Craen, A.J.M., Wright, M.J., Launer, L.J., Schumann, G., Fornage, M., Franke, B., Debette, S., Medland, S.E., Ikram, M.A., \& Thompson, P.M. (2016) Novel genetic loci underlying human intracranial volume identified through genome-wide association. Nat. Neurosci., 19, 1569-1582.

Annett, M. (1970) A classification of hand preference by association analysis. Br. J. Psychol., 61, 303-321.

Bhatt, R.R., Gupta, A., Labus, J.S., Zeltzer, L.K., Tsao, J.C., Shulman, R.J., \& Tillisch, K. (2018) Altered Brain Structure and Functional Connectivity and its Relation to Pain Perception in Girls with Irritable Bowel Syndrome. Psychosom. Med.,.

Chirachariyavej, T., Ouyswat, K., Sanggarnjanavanich, S., Tiensuwan, M., Peonim, V., \& Sirikulchayanonta, V. (2006) Normal internal organ weight of Thai adults correlated to body length and body weight. J. Med. Assoc. Thai., 89, 1702-1712.

Cohen, J. (1992) A Power Primer. Psychol. Bull., 112, 155-159.

Costafreda, S.G., Dinov, I.D., Tu, Z., Shi, Y., Liu, C.-Y., Kloszewska, I., Mecocci, P., Soininen, H., Tsolaki, M., Vellas, B., Wahlund, L.-O., Spenger, C., Toga, A.W., Lovestone, S., \& Simmons, A. (2011) Automated hippocampal shape analysis predicts the onset of dementia in mild cognitive impairment. Neuroimage, 56, 212-219.

Destrieux, C., Fischl, B., Dale, A., \& Halgren, E. (2010) Automatic parcellation of human cortical gyri and sulci using standard anatomical nomenclature. Neuroimage, 53, 1-15.

Erickson, K.I., Voss, M.W., Prakash, R.S., Basak, C., Szabo, A., Chaddock, L., Kim, J.S., Heo, S., Alves, H., White, S.M., Wojcicki, T.R., Mailey, E., Vieira, V.J., Martin, S.A., Pence, B.D., Woods, J.A., McAuley, E., \& Kramer, A.F. (2011) Exercise training increases size of hippocampus and improves memory. Proc. Natl. Acad. Sci. U. S. A., 108, 3017-3022.

Fischl, B., Salat, D.H., Busa, E., Albert, M., Dieterich, M., Haselgrove, C., van der Kouwe, A., Killiany, R., Kennedy, D., Klaveness, S., Montillo, A., Makris, N., Rosen, B., \& Dale, A.M. (2002) Whole brain segmentation: automated labeling of neuroanatomical structures in the human brain. Neuron, 33, 341-355.

Fischl, B., Salat, D.H., van der Kouwe, A.J., Makris, N., Segonne, F., Quinn, B.T., \& Dale, A.M. (2004) Sequence-independent segmentation of magnetic resonance images.

Neuroimage, 23 Suppl 1, S69-S84.

Fischl, B., van der Kouwe, A., Destrieux, C., Halgren, E., Segonne, F., Salat, D.H., Busa, E., 
Seidman, L.J., Goldstein, J., Kennedy, D., Caviness, V., Makris, N., Rosen, B., \& Dale, A.M. (2004) Automatically parcellating the human cerebral cortex. Cereb. Cortex, 14, $11-22$.

Garby, L., Lammert, O., Kock, K.F., \& Thobo-Carlsen, B. (1993) Weights of brain, heart, liver, kidneys, and spleen in healthy and apparently healthy adult Danish subjects. Am. J. Hum. Biol., 5, 291-296.

Gaser, C., Franke, K., Klöppel, S., Koutsouleris, N., Sauer, H., \& Alzheimer's, Disease Neuroimaging Initiative (2013) BrainAGE in Mild Cognitive Impaired Patients: Predicting the Conversion to Alzheimer's Disease. PLoS One, 8, e67346.

Grömping, U. (2006) Relative Importance for Linear Regression in R: The Package relaimpo. J. Stat. Softw., 17, 1-27.

Hänggi, J., Fövenyi, L., Liem, F., Meyer, M., \& Jäncke, L. (2014) The hypothesis of neuronal interconnectivity as a function of brain size-a general organization principle of the human connectome. Front. Hum. Neurosci., 8, 915.

Hartmann, P., Ramseier, A., Gudat, F., Mihatsch, M.J., \& Polasek, W. (1994) [Normal weight of the brain in adults in relation to age, sex, body height and weight]. Pathologe, 15, 165-170.

Haug, H. (1984) Der Einfluß der säkularen Akzeleration auf das Hirngewicht des Menschen und dessen Änderung während der Alterung. Gegenbaurs Morphol. Jahrb., 130, 481500.

Hedman, A.M., van Haren, N.E.M., Schnack, H.G., Kahn, R.S., \& Hulshoff Pol, H.E. (2012) Human brain changes across the life span: a review of 56 longitudinal magnetic resonance imaging studies. Hum. Brain Mapp., 33, 1987-2002.

Heymsfield, S.B., Chirachariyavej, T., Rhyu, I.J., Roongpisuthipong, C., Heo, M., \& Pietrobelli, A. (2009) Differences between brain mass and body weight scaling to height: potential mechanism of reduced mass-specific resting energy expenditure of taller adults. J. Appl. Physiol., 106, 40-48.

Heymsfield, S.B., Gallagher, D., Mayer, L., Beetsch, J., \& Pietrobelli, A. (2007) Scaling of human body composition to stature: new insights into body mass index. Am. J. Clin. Nutr., 86, 82-91.

Hirsiger, S., Koppelmans, V., Mérillat, S., Liem, F., Erdeniz, B., Seidler, R.D., \& Jäncke, L. (2015) Structural and functional connectivity in healthy aging: Associations for cognition and motor behavior. Hum. Brain Mapp.,.

Ho, K.C., Roessmann, U., Straumfjord, J.V., \& Monroe, G. (1980) Analysis of brain weight. II. Adult brain weight in relation to body height, weight, and surface area. Arch. Pathol. Lab. Med., 104, 640-645.

Holloway, R.L. (1980) Within-species brain-body weight variability: a reexamination of the Danish data and other primate species. Am. J. Phys. Anthropol., 53, 109-121.

Jäncke, L. (2009) The plastic human brain. Restor. Neurol. Neurosci., 27, 521-538.

Jäncke, L., Liem, F., \& Merillat, S. (2019) Scaling of brain compartments to brain size. Neuroreport, 30, 573-579.

Jäncke, L., Mérillat, S., Liem, F., \& Hänggi, J. (2015) Brain size, sex, and the aging brain. Hum. Brain Mapp., 36, 150-169.

Jäncke, L., Staiger, J.F., Schlaug, G., Huang, Y., \& Steinmetz, H. (1997) The relationship between corpus callosum size and forebrain volume. Cereb. Cortex, 7, 48-56.

Jäncke, L. \& Steinmetz, H. (1998) Brain size: a possible source of interindividual variability in corpus callosum morphology [WWW Document]. The role of the human corpus callosum in sensory motor integration: anatomy, physiology, and behavior; individual differences and clinical applications,. URL http://cogprints.org/86/2/nato-cc.pdf

Jerison, H.J. (1979) The evolution of diversity in brain size. In Hahn, M.E., Jensen, C., \& C.,

This article is protected by copyright. All rights reserved. 
D.B. (eds), Development and Evolution in Brain Size. Academic Press, New York, pp. 29-57.

Jurgens, H.W., Aune, I.A., \& Pieper, U. (1990) International Data on Anthropometry. International Labour Office, Geneva, Switzerland.

Keller, M.C., Garver-Apgar, C.E., Wright, M.J., Martin, N.G., Corley, R.P., Stallings, M.C., Hewitt, J.K., \& Zietsch, B.P. (2013) The genetic correlation between height and IQ: shared genes or assortative mating? PLoS Genet., 9, e1003451.

Koh, I., Lee, M.S., Lee, N.J., Park, K.W., Kim, K.H., Kim, H., \& Rhyu, I.J. (2005) Body size effect on brain volume in Korean youth. Neuroreport, 16, 2029-2032.

Krauth, J. (1988) Distribution-Free Statistics: An Application-Oriented Approach. Elsevier Science Ltd.

Kurth, F., Jancke, L., \& Luders, E. (2017) Sexual dimorphism of Broca's region: More gray matter in female brains in Brodmann areas 44 and 45. J. Neurosci. Res., 95, 626-632.

Lai, C.-Q. (2006) How much of human height is genetic and how much is due to nutrition. Sci. Am., 11.

Luders, E., Narr, K.L., Thompson, P.M., Rex, D.E., Jancke, L., Steinmetz, H., \& Toga, A.W. (2004) Gender differences in cortical complexity. Nat. Neurosci., 7, 799-800.

Luders, E., Narr, K.L., Thompson, P.M., Rex, D.E., Woods, R.P., Deluca, H., Jancke, L., \& Toga, A.W. (2006) Gender effects on cortical thickness and the influence of scaling. Hum. Brain Mapp., 27, 314-324.

Luders, E., Narr, K.L., Thompson, P.M., \& Toga, A.W. (2009) Neuroanatomical Correlates of Intelligence. Intelligence, 37, 156-163.

Luders, E., Narr, K.L., Thompson, P.M., Woods, R.P., Rex, D.E., Jancke, L., Steinmetz, H., $\&$ Toga, A.W. (2005) Mapping cortical gray matter in the young adult brain: effects of gender. Neuroimage, 26, 493-501.

Lüders, E., Steinmetz, H., \& Jäncke, L. (2002) Brain size and grey matter volume in the healthy human brain. Neuroreport, 13, 2371-2374.

Lukies, M.W., Watanabe, Y., Tanaka, H., Takahashi, H., Ogata, S., Omura, K., Yorifuji, S., Tomiyama, N., \& Osaka University Twin Research Group (2017) Heritability of brain volume on MRI in middle to advanced age: A twin study of Japanese adults. PLoS One, 12, e0175800.

Madhyastha, T., Mérillat, S., Hirsiger, S., Bezzola, L., Liem, F., Grabowski, T., \& Jäncke, L. (2014) Longitudinal Reliability of Tract-Based Spatial Statistics in Diffusion Tensor Imaging.

Marioni, R.E., Batty, G.D., Hayward, C., Kerr, S.M., Campbell, A., Hocking, L.J., Generation Scotland, Porteous, D.J., Visscher, P.M., \& Deary, I.J. (2014) Common genetic variants explain the majority of the correlation between height and intelligence: the generation Scotland study. Behav. Genet., 44, 91-96.

Mattson, M.P., Chan, S.L., \& Duan, W. (2002) Modification of brain aging and neurodegenerative disorders by genes, diet, and behavior. Physiol. Rev., 82, 637-672.

Miskolczi, C., Halász, J., \& Mikics, É. (2018) Changes in neuroplasticity following early-life social adversities: the possible role of brain-derived neurotrophic factor. Pediatr. Res.,.

Münte, T.F., Altenmüller, E., \& Jäncke, L. (2002) The musician's brain as a model of neuroplasticity. Nat. Rev. Neurosci., 3, 473-478.

NASA Reference Publication (1978) Anthropometric Source Book. Volume I: Anthropometry for Designers. Anthropology Research Project, Yellow Springs, Ohio:

NCD Risk Factor Collaboration (NCD-RisC) (2016) A century of trends in adult human height. Elife, 5.

Nopoulos, P., Flaum, M., O’Leary, D., \& Andreasen, N.C. (2000) Sexual dimorphism in the human brain: evaluation of tissue volume, tissue composition and surface anatomy using

This article is protected by copyright. All rights reserved. 
magnetic resonance imaging. Psychiatry Res., 98, 1-13.

Oltmanns, J., Godde, B., Winneke, A.H., Richter, G., Niemann, C., Voelcker-Rehage, C., Schömann, K., \& Staudinger, U.M. (2017) Don't Lose Your Brain at Work - The Role of Recurrent Novelty at Work in Cognitive and Brain Aging. Front. Psychol., 8, 117.

Paigen, B., Goldman, L.R., Magnant, M.M., Highland, J.H., \& Steegmann, A.T., Jr (1987) Growth of children living near the hazardous waste site, Love Canal. Hum. Biol., 59, 489-508.

Pakkenberg, H. \& Voigt, J. (1964) Brain weight of the Danes: Forensic material. Acta Anat., 56, 297-307.

Pascual-Leone, A., Amedi, A., Fregni, F., \& Merabet, L.B. (2005) The plastic human brain cortex. Annu. Rev. Neurosci., 28, 377-401.

Passingham, R.E. (1979) Brain size and intelligence in man. Brain Behav. Evol., 16, 253270.

Peters, M., Jäncke, L., Staiger, J.F., Schlaug, G., Huang, Y., \& Steinmetz, H. (1998) Unsolved problems in comparing brain sizes in Homo sapiens. Brain Cogn., 37, 254 285.

Posthuma, D., de Geus, E.J., Neale, M.C., Hulshoff Pol, H.E., Baaré WEC, Kahn, R.S., \& Boomsma, D. (2000) Multivariate genetic analysis of brain structure in an extended twin design. Behav. Genet., 30, 311-319.

Raz, N., Gunning, F.M., Head, D., Dupuis, J.H., McQuain, J., Briggs, S.D., Loken, W.J., Thornton, A.E., \& Acker, J.D. (1997) Selective aging of the human cerebral cortex observed in vivo: differential vulnerability of the prefrontal gray matter. Cereb. Cortex, 7, 268-282.

R Core Team (2013) R: A Language and Environment for Statistical Computing.

Reardon, P.K., Seidlitz, J., Vandekar, S., Liu, S., Patel, R., Park, M.T.M., Alexander-Bloch, A., Clasen, L.S., Blumenthal, J.D., Lalonde, F.M., Giedd, J.N., Gur, R.C., Gur, R.E., Lerch, J.P., Chakravarty, M.M., Satterthwaite, T.D., Shinohara, R.T., \& Raznahan, A. (2018) Normative brain size variation and brain shape diversity in humans. Science, 360, 1222-1227.

Ringo, J.L., Doty, R.W., Demeter, S., \& Simard, P.Y. (1994) Time is of the essence: a conjecture that hemispheric specialization arises from interhemispheric conduction delay. Cereb. Cortex, 4, 331-343.

Roth, G. \& Dicke, U. (2005) Evolution of the brain and intelligence. Trends Cogn. Sci., 9, $250-257$.

Russ, T.C., Kivimäki, M., Starr, J.M., Stamatakis, E., \& Batty, G.D. (2014) Height in relation to dementia death: individual participant meta-analysis of $18 \mathrm{UK}$ prospective cohort studies. Br. J. Psychiatry, 205, 348-354.

Sammallahti, S., Pyhälä, R., Lahti, M., Lahti, J., Pesonen, A.-K., Heinonen, K., Hovi, P., Eriksson, J.G., Strang-Karlsson, S., Andersson, S., Järvenpää, A.-L., Kajantie, E., \& Räikkönen, K. (2014) Infant growth after preterm birth and neurocognitive abilities in young adulthood. J. Pediatr., 165, 1109-1115.e3.

Silventoinen, K., Iacono, W.G., Krueger, R., \& McGue, M. (2012) Genetic and environmental contributions to the association between anthropometric measures and iq: a study of Minnesota twins at age 11 and 17. Behav. Genet., 42, 393-401.

Skullerud, K. (1985) Variations in the size of the human brain. Influence of age, sex, body length, body mass index, alcoholism, Alzheimer changes, and cerebral atherosclerosis. Acta Neurol. Scand. Suppl., 102, 1-94.

Spann, W. \& Dustmann, H.O. (1965) [Weight of the human brain and its dependence on age, body length, cause of death and occupation]. Dtsch. Z. Gesamte Gerichtl. Med., 56, 299-317.

This article is protected by copyright. All rights reserved. 
Sturm, W., Horn, W., \& Willmes, K. (1993) Leistungsprüfsystem Für 50-90jährige:(LPS 50+); Handanweisung. Hogrefe, Verlag für Psychologie.

Sudfeld, C.R., McCoy, D.C., Danaei, G., Fink, G., Ezzati, M., Andrews, K.G., \& Fawzi, W.W. (2015) Linear growth and child development in low- and middle-income countries: a meta-analysis. Pediatrics, 135, e1266-e1275.

Sudfeld, C.R., McCoy, D.C., Fink, G., Muhihi, A., Bellinger, D.C., Masanja, H., Smith, E.R., Danaei, G., Ezzati, M., \& Fawzi, W.W. (2015) Malnutrition and Its Determinants Are Associated with Suboptimal Cognitive, Communication, and Motor Development in Tanzanian Children. J. Nutr., 145, 2705-2714.

Taki, Y., Hashizume, H., Sassa, Y., Takeuchi, H., Asano, M., Asano, K., Kotozaki, Y., Nouchi, R., Wu, K., Fukuda, H., \& Kawashima, R. (2012) Correlation among body height, intelligence, and brain gray matter volume in healthy children. Neuroimage, $\mathbf{5 9}$, 1023-1027.

Valizadeh, S.A., Hänggi, J., Mérillat, S., \& Jäncke, L. (2017) Age prediction on the basis of brain anatomical measures. Hum. Brain Mapp., 38, 997-1008.

Valizadeh, S.A., Liem, F., Mérillat, S., Hänggi, J., \& Jäncke, L. (2018) Identification of individual subjects on the basis of their brain anatomical features. Sci. Rep., 8, 5611.

Voelcker-Rehage, C., Godde, B., \& Staudinger, U.M. (2010) Physical and motor fitness are both related to cognition in old age. Eur. J. Neurosci., 31, 167-176.

Voss, M.W., Heo, S., Prakash, R.S., Erickson, K.I., Alves, H., Chaddock, L., Szabo, A.N., Mailey, E.L., Wójcicki, T.R., White, S.M., Gothe, N., McAuley, E., Sutton, B.P., \& Kramer, A.F. (2013) The influence of aerobic fitness on cerebral white matter integrity and cognitive function in older adults: results of a one-year exercise intervention. Hum. Brain Mapp., 34, 2972-2985.

Vuoksimaa, E., Panizzon, M.S., Franz, C.E., Fennema-Notestine, C., Hagler, D.J., Jr, Lyons, M.J., Dale, A.M., \& Kremen, W.S. (2018) Brain structure mediates the association between height and cognitive ability. Brain Struct. Funct., 223, 3487-3494.

Wickett, J.C., Vernon, P.A., \& Lee, D.H. (1994) In vivo brain size, head perimeter, and intelligence in a sample of healthy adult females. Pers. Individ. Dif., 16, 831-838.

Widdowson, E.M. (1951) Mental contentment and physical growth. Lancet, 1, 1316-1318.

Willerman, L., Schultz, R., Neal Rutledge, J., \& Bigler, E.D. (1991) In vivo brain size and intelligence. Intelligence, 15, 223-228.

Witelson, S.F., Beresh, H., \& Kigar, D.L. (2006) Intelligence and brain size in 100 postmortem brains: sex, lateralization and age factors. Brain, 129, 386-398.

Zatorre, R.J., Fields, R.D., \& Johansen-Berg, H. (2012) Plasticity in gray and white: neuroimaging changes in brain structure during learning. Nat. Neurosci., 15, 528-536.

Ziegler, G., Dahnke, R., Jäncke, L., Yotter, R.A., May, A., \& Gaser, C. (2012) Brain structural trajectories over the adult lifespan. Hum. Brain Mapp., 33, 2377-2389.

This article is protected by copyright. All rights reserved. 


\section{Figure caption}

Figure 1: Scatterplots for body height and the 10 brain metrics. The brain metrics are corrected for Age and Sex. For more convenient annotation of some brain metrics we have introduced shorter acronyms than in the main text (SBCOGM: SubCortGrayVol; CBGM: CerebellumCortex; CBWM: CerebellumWhiteMatter).

\section{Table captions}

Table 1: Means and standard deviations for Age (in years), body height $(\mathrm{BH}$; in $\mathrm{cm})$, education in years (Years of education), and IQ for the subjects broken down for sex. In addition, results of t-tests (Welch Two Sample t-test) comparing men and women with the tstatistic, degrees of freedom, exact p-values, and Cohen's d are shown.

Table 2: Means and standard deviations for all brain metrics broken down for sex. Volumes (ICV, BV, CortexVol, SubCortGrayVol, NAWM, WMH, CerebellumWhiteMatter, CerebellumCortex) are shown in $\mathrm{cm}^{3}, \mathrm{CT}$ in $\mathrm{mm}$, and CSA in $\mathrm{cm}^{2}$. In addition, results of $\mathrm{t}$ tests (Welch Two Sample t-test) comparing men and women with the t-statistic, degrees of freedom (df), exact p-values, and Cohen's d are shown.

Table 3: Correlations between the different brain metrics. For more convenient annotation of some brain metrics we have introduced shorter acronyms than in the main text (SBCOGM: SubCortGrayVol; CBGM: CerebellumCortex; CBWM: CerebellumWhiteMatter).

Table 4: Results of the factor analysis indicated as loadings of the brain metrics. RC1-RC6 are the rotated factors ordered according to the explained variance. Strong loadings $(>0.8)$ are indicated in bold letters.

Table 5: Summary of the multiple regression analysis with $\mathrm{Sex}, \mathrm{BH}$, Age, and the interactions between Sex x Age, BH x Sex, and $\mathrm{BH} \times$ Age. The influence of each independent variable is indicated as percent of explained variance $\left(\mathrm{R}^{2} * 100\right)$. These variance estimates are derived from the $\operatorname{lmg}$ based estimation of the relative contribution of each predictor. Variance estimates between 1-9\% are classified as small effects while variance estimates between $9-25 \%$ are considered as medium size effects. Indicated are also the rotated factors on which the particular brain metrics loads. 
Table 6: Pearson correlations between $\mathrm{BH}$ and the different brain metrics. The brain metrics are corrected for Age and Sex. Indicated are the correlation estimates (r), the associated pvalues, and the $95 \%$ confidence intervals for the correlation estimates. "Small" correlations according to Cohen's effect size categorization $(r=0.1-0.3)$ are indicated in bold. Indicated are also the rotated factors on which the particular brain metrics loads.

This article is protected by copyright. All rights reserved. 


\section{Tables}

Table 1: Means and standard deviations for Age (in years), body height ( $\mathrm{BH}$; in $\mathrm{cm}$ ), education in years (Years of education), and IQ for the subjects broken down for sex. In addition, results of t-tests (Welch Two Sample t-test) comparing men and women with the tstatistic, degrees of freedom, exact p-values, and Cohen's d are shown.

\begin{tabular}{|l|l|l|l|l|l|l|l|l|l|}
\hline \multicolumn{1}{l}{ Women } & \multicolumn{2}{l}{ Men } & \multicolumn{2}{l|}{} \\
\hline & Mean & Sd & Mean & Sd & t & df & p & d \\
\hline Age & 70.5 & 5.1 & 71.0 & 5.2 & -0.65 & 213.9 & 0.51579 & 0.09 \\
\hline BH & 164.7 & 5.9 & 175.9 & 6.0 & -13.88 & 214.0 & $1.136879 \mathrm{e}-31$ & 1.89 \\
\hline Years of education & 13.4 & 3.0 & 15.8 & 3.8 & -4.93 & 191.2 & $1.743360 \mathrm{e}-06$ & 0.67 \\
\hline IQ & 119.7 & 5.9 & 122.0 & 7.3 & -3.49 & 190.7 & 0.00059 & 0.48 \\
\hline
\end{tabular}

${ }^{1}$ : IQ could be slightly overestimated since the subjects mostly fall exactly in the age range between two age categories (55-69 years and 70-90 years). We have used the normalization for the age category 70-90 years.

This article is protected by copyright. All rights reserved. 
Table 2: Means and standard deviations for all brain metrics broken down for sex. Volumes (ICV, BV, CortexVol, SubCortGrayVol, NAWM, WMH, CerebellumWhiteMatter,

CerebellumCortex) are shown in $\mathrm{cm}^{3}, \mathrm{CT}$ in $\mathrm{mm}$, and CSA in $\mathrm{cm}^{2}$. In addition, results of $\mathrm{t}-$ tests (Welch Two Sample t-test) comparing men and women with the t-statistic, degrees of freedom (df), exact p-values, and Cohen's d are shown.

\begin{tabular}{|c|c|c|c|c|c|c|c|c|}
\hline & $\begin{array}{l}\text { Wome } \\
\mathbf{n}\end{array}$ & & Men & & & & & \\
\hline & m & sd & m & sd & $\mathbf{t}$ & df & $\mathbf{p}$ & d \\
\hline ICV & 1336.6 & 196.9 & 1586.4 & 201.3 & -9.22 & 214.0 & $2.883902 \mathrm{e}-17$ & 1.25 \\
\hline BV & 981.8 & 76.8 & 1088.8 & 90.4 & -9.38 & 209.7 & $1.115562 \mathrm{e}-17$ & 1.28 \\
\hline CSA & 1345.4 & 98.9 & 1481.5 & 111.3 & -9.50 & 211.9 & $4.543555 e-18$ & 1.29 \\
\hline CT & 2.3 & 0.1 & 2.3 & 0.1 & 1.04 & 214.0 & 0.30131 & 0.14 \\
\hline CortexVol & 404.2 & 32.0 & 444.3 & 32.8 & -9.09 & 214.0 & $6.788876 e-17$ & 1.24 \\
\hline SubCortGrayVol & 51.1 & 4.0 & 55.8 & 4.1 & -8.55 & 213.9 & $2.310621 \mathrm{e}-15$ & 1.16 \\
\hline NAWM & 402.5 & 41.7 & 452.4 & 52.6 & -7.72 & 205.0 & $4.930521 \mathrm{e}-13$ & 1.05 \\
\hline WMH & 3.6 & 5.1 & 3.3 & 3.1 & 0.61 & 174.7 & 0.54230 & 0.08 \\
\hline CerebellumWhiteMatter & 26.5 & 3.5 & 27.8 & 4.1 & -2.50 & 210.6 & 0.01316 & 0.34 \\
\hline CerebellumCortex & 93.0 & 8.4 & 104.0 & 9.7 & -8.94 & 210.2 & $<0.00001$ & 1.22 \\
\hline
\end{tabular}

This article is protected by copyright. All rights reserved. 
Table 3: Correlations between the different brain metrics. For more convenient annotation of some brain metrics we have introduced shorter acronyms than in the main text (SBCOGM: SubCortGrayVol; CBGM: CerebellumCortex; CBWM: CerebellumWhiteMatter).

\begin{tabular}{|c|c|c|c|c|c|c|c|c|c|}
\hline Brain metric & BV & CSA & CT & CortexVol & SBCOGM & NAWM & WMH & CBWM & CBGM \\
\hline ICV & 0.62 & 0.63 & -0.12 & 0.59 & 0.57 & 0.52 & 0.26 & 0.30 & 0.51 \\
\hline BV & & 0.93 & 0.15 & 0.91 & 0.87 & 0.95 & -0.08 & 0.59 & 0.71 \\
\hline CSA & & & -0.06 & 0.90 & 0.78 & 0.86 & -0.03 & 0.44 & 0.59 \\
\hline CT & & & & 0.33 & 0.16 & 0.01 & -0.25 & 0.10 & 0.16 \\
\hline CortexVol & & & & & 0.78 & 0.76 & -0.09 & 0.41 & 0.63 \\
\hline SBCOGM & & & & & & 0.80 & -0.02 & 0.54 & 0.65 \\
\hline NAWM & & & & & & & -0.15 & 0.57 & 0.58 \\
\hline WMH & & & & & & & & -0.06 & -0.08 \\
\hline CBWM & & & & & & & & & 0.57 \\
\hline
\end{tabular}

This article is protected by copyright. All rights reserved. 
Table 4: Results of the factor analysis indicated as loadings of the brain metrics. RC1-RC6 are the rotated factors ordered according to the explained variance. Strong loadings $(>0.8)$ are indicated in bold letters.

\begin{tabular}{|l|l|l|l|l|l|l|}
\hline & RC1 & RC4 & RC3 & RC2 & RC6 & RC5 \\
\hline Explained Variance & 44.10 & 11.50 & 11.30 & 10.40 & 9.40 & 9.40 \\
\hline ICV & 0.45 & 0.08 & -0.09 & 0.18 & $\mathbf{0 . 8 5}$ & 0.17 \\
\hline BV & $\mathbf{0 . 9 1}$ & 0.26 & 0.08 & -0.04 & 0.19 & 0.23 \\
\hline CSA & $\mathbf{0 . 9 3}$ & 0.08 & -0.11 & -0.01 & 0.22 & 0.15 \\
\hline CT & 0.06 & 0.04 & $\mathbf{0 . 9 8}$ & -0.12 & -0.06 & 0.05 \\
\hline CortexVol & $\mathbf{0 . 8 7}$ & 0.04 & 0.30 & -0.03 & 0.25 & 0.20 \\
\hline SubCortGrayVol & $\mathbf{0 . 8 1}$ & 0.26 & 0.12 & 0.06 & 0.15 & 0.24 \\
\hline NAWM & $\mathbf{0 . 8 9}$ & 0.31 & -0.08 & -0.13 & 0.10 & 0.09 \\
\hline WMH & -0.06 & -0.02 & -0.12 & $\mathbf{0 . 9 8}$ & 0.11 & -0.02 \\
\hline $\begin{array}{l}\text { CerebellumWhiteM } \\
\text { atter }\end{array}$ & 0.33 & $\mathbf{0 . 9 1}$ & 0.04 & -0.02 & 0.06 & 0.20 \\
\hline CerebellumCortex & 0.44 & 0.27 & 0.08 & -0.04 & 0.18 & $\mathbf{0 . 8 3}$ \\
\hline
\end{tabular}

This article is protected by copyright. All rights reserved. 
Table 5: Summary of the multiple regression analysis with Sex, BH, Age, and the interactions between Sex x Age, BH x Sex, and BH x Age. The influence of each independent variable is indicated as percent of explained variance $\left(R^{2} * 100\right)$. These variance estimates are derived from the $l m g$ based estimation of the relative contribution of each predictor. Variance estimates between 1-9\% are classified as small effects while variance estimates between $9-25 \%$ are considered as medium size effects. Indicated are also the rotated factors on which the particular brain metrics loads.

\begin{tabular}{|l|l|l|l|l|l|l|}
\hline & BH & Sex & Age & Sex:Age & BH:Sex & BH:Age \\
\hline ICV (RC5) & 5.62 & 22.26 & 4.62 & 0.11 & 0.39 & 0.63 \\
\hline BV (RC1) & 12.44 & 19.45 & 9.26 & 0.12 & 0.23 & 0.02 \\
\hline CSA (RC1) & 11.49 & 20.21 & 5.69 & 0.03 & 0.46 & 0.02 \\
\hline CT (RC3) & 0.22 & 0.60 & 11.89 & 0.17 & 0.40 & 0.49 \\
\hline CortexVol (RC1) & 12.42 & 18.41 & 8.58 & 0.16 & 0.08 & 0.18 \\
\hline SubCortGrayVol (RC1) & 8.76 & 18.13 & 7.93 & 0.44 & 0.30 & 0.12 \\
\hline NAWM (RC1) & 9.45 & 14.58 & 9.96 & 0.41 & 0.41 & 0.07 \\
\hline WMH (RC4) & 0.48 & 0.14 & 15.47 & 0.40 & 0.84 & 0.24 \\
\hline CerebellumCortex (RC6) & 10.71 & 18.33 & 4.12 & 0.25 & 0.04 & 0.09 \\
\hline $\begin{array}{l}\text { CerebellumWhiteMatter } \\
\text { (RC2) }\end{array}$ & 2.18 & 1.66 & 2.63 & 0.82 & 0.03 & 0.37 \\
\hline
\end{tabular}

This article is protected by copyright. All rights reserved. 
Table 6: Pearson correlations between $\mathrm{BH}$ and the different brain metrics. The brain metrics are corrected for Age and Sex. Indicated are the correlation estimates (r), the associated pvalues, and the $95 \%$ confidence intervals for the correlation estimates. "Small" correlations according to Cohen's effect size categorization $(r=0.1-0.3)$ are indicated in bold. Indicated are also the rotated factors on which the particular brain metrics loads.

\begin{tabular}{|c|c|c|c|c|}
\hline & $r$ & $\mathbf{p}$ & $\mathrm{ci}_{2.5 \%}$ & $\mathrm{Ci}_{97.5 \%}$ \\
\hline ICV (RC5) & -0.06 & 0.3616 & -0.19 & 0.07 \\
\hline BV (RC1) & 0.19 & 0.0056 & 0.06 & 0.31 \\
\hline CSA (RC1) & 0.16 & 0.0210 & 0.02 & 0.28 \\
\hline CT (RC3) & 0.09 & 0.1890 & -0.04 & 0.22 \\
\hline CortexVol (RC1) & 0.19 & 0.0041 & 0.06 & 0.32 \\
\hline SubCortGrayVol (RC1) & 0.11 & 0.1033 & -0.02 & 0.24 \\
\hline NAWM (RC1) & 0.16 & 0.0178 & 0.03 & 0.29 \\
\hline WMH (RC4) & -0.08 & 0.2335 & -0.21 & 0.05 \\
\hline CerebellumCortex (RC6) & 0.15 & 0.0258 & 0.02 & 0.28 \\
\hline CerebellumWhiteMatter (RC2) & 0.11 & 0.1237 & -0.03 & 0.24 \\
\hline
\end{tabular}

This article is protected by copyright. All rights reserved. 

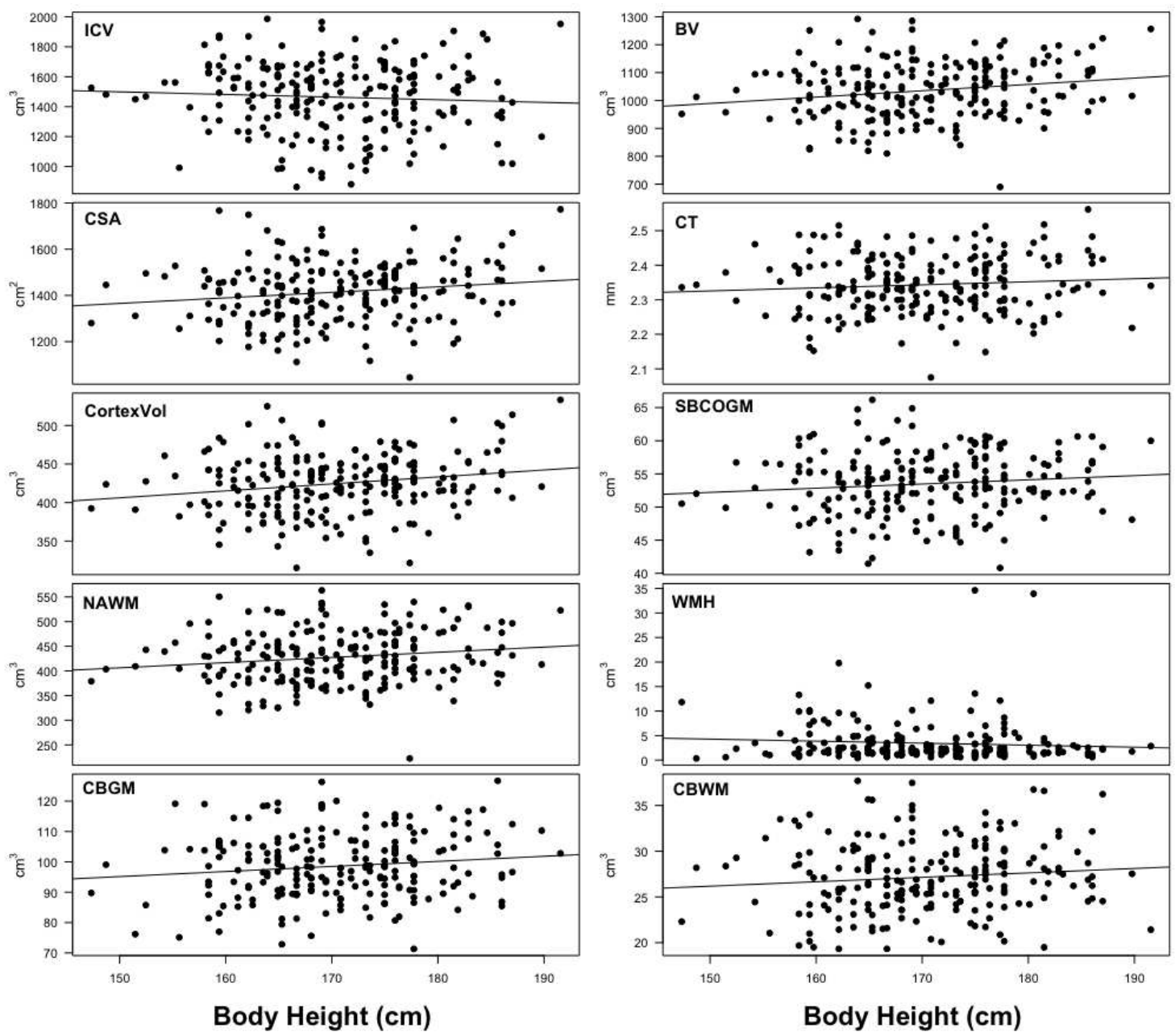

This article is protected by copyright. All rights reserved. 